\title{
DYE DILUTION CURVES IN CONGENITAL HEART DISEASE
}

\author{
BY \\ C. OAKLEY, S. TAYLOR, D. WILCKEN, A. HOLLMAN, J. F. GOODWIN, \\ AND J. SHILLINGFORD
}

From the Department of Medicine, Postgraduate Medical School of London

Received October 21, 1959

The need for methods capable of detecting and estimating roughly the size of congenital cardiac defects has increased with the recent rapid developments in cardiac surgery. The application of dye dilution technique to the study of these patients is largely due to the extensive work of Wood and his colleagues at the Mayo Clinic since 1950. However, although the development of these techniques represents a most valuable addition to the methods available for investigation, they are perhaps more elaborate than is possible in many smaller centres engaged in the diagnosis of congenital heart disease.

Recording the passage of dye through the pinna of the transilluminated ear by a photoelectric cell and recording apparatus offers the advantage of simplicity, but has not found universal favour because of technical difficulties and lack of a suitable dye. With the development of high-stability electronic amplifiers and recorders and the introduction of Coomassie blue, we have found this method to be satisfactory for qualitative investigations. The purpose of our paper is to describe the results of this method used at the time of right heart catheterization in a series of patients with congenital heart disease, and to assess its value in clinical practice.

\section{Material AND Methods}

Sixty-eight unselected patients with suspected congenital heart disease were studied at rest by means of right heart catheterization (Table I). Pressures were recorded by electromanometers and direct writing recorder. Arterial and venous blood samples were estimated for oxygen saturation by a modification of Gatman's spectrophotometric technique (Wade et al., 1953) or, if the samples had been taken after injecting dye, they were estimated by the Haldane method. The ear oximeter was based on the design of Wood and Geraci (1949) and has been used in previous work from this laboratory (Korner and Shillingford, 1955; Taylor and Shillingford, 1959).

The success of the technique depends to a large extent on the recording apparatus and the design of this has presented considerable difficulty owing to the very small changes in current in the photo-electric cell during the passage of the blue dye through the ear. We have used the Cambridge earpiece and chopper amplifier, feeding either into an Evershed and Vignole 0-1 m.a. recorder or a Cambridge recorder (Fig. 1). This combination gives the high amplification and stability necessary for this method of recording. Amplifiers and recorders used in electrocardiographic or pressure recording systems have proved quite unsuitable.

The sensitivity of the amplification system was arranged to give a maximum deflection of $5-7 \mathrm{~cm}$. at the peak of the dilution curve, the sensitivity chosen being the highest at which a stable base line could be obtained with the patient breathing room air. After recording pressures from a chamber of the heart, blood samples were taken for determination of blood oxygen saturation and the dye was then immediately injected as rapidly as possible. The site of the injection was checked by screening and manometry before and after the injection. The dose range of Coomassie blue was usually of the order of 0.5 to $1.0 \mathrm{mg}$. per $\mathrm{Kg}$. body weight per injection and up to 10 injections of dye have been given without any untoward reactions.

In some patients cardiac catheterization was followed by selective angiocardiography. In many the diagnosis was confirmed at operation. In some patients dye dilution curves were performed in the operating room immediately before and after surgical repair of the cardiac defect. 


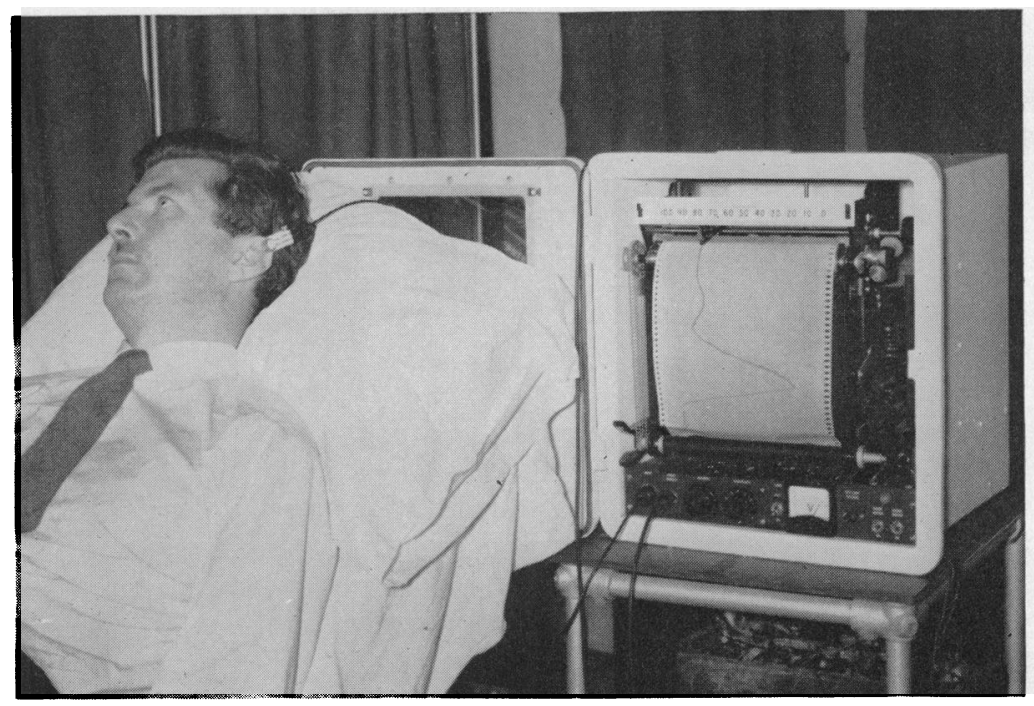

Fig. 1.-The earpiece and the recorder.

The Normal Dye Dilution Curve

Fig. 2A shows a dye dilution curve obtained by this method from a patient without heart disease. The time from the injection to the beginning of the upstroke of the curve represents the passage of the dye from the site of injection through the circulation to the ear. Normally from the right atrium to the ear the time is approximately $10 \mathrm{sec}$. (Carter et al., 1959). It varies with the length of passage of the dye, the volume of fluid through which it flows, and the cardiac output. The upstroke of the curve rises rapidly to the peak concentration and the downslope is exponential in form. The recirculation curve appears on the descending limb of the downslope, usually as it approaches the base line, and in some cases a second recirculation curve may be seen before complete mixing of the dye occurs and the final base line settles at a level above that of the initial base line. The difference of the levels represents the final concentration of dye in the blood.

Left-to-Right Shunts. The effect of a left-to-right shunt on the form of the dye curve is shown in Fig. 2B. The appearance time is within normal limits or slightly shortened. The upstroke is rapid and the peak tends to be low because some dye is lost in pulmonary recirculation, and for the same reason the exponential fall off is replaced by a series of small curves due to pulmonary recirculation. These may be of such a size as not to be seen individually although the overall effect is to prolong the downslope.

The higher the ratio of pulmonary to systemic blood flow, the lower the peak of the curve and the slower the fall off. To obtain a satisfactory curve in cases with large shunts a larger injection dose is needed.

A rough quantitative estimate of the size of the shunt can be obtained by the slowness of the fall off in proportion to the steepness of the upstroke (skewing). Fig. 3 shows the appearance of dye curves with left-to-right shunts of various magnitudes. No attempt has been made to make a more accurate quantitative estimate in this study.

Right-to-Left Shunts. The distortion produced by a right-to-left shunt is illustrated in Fig. 2C. Dye bypassing the lungs arrives early at the ear so that the appearance time is short (3-5 sec.). Dye traversing the normal route via the pulmonary circulation arrives at the ear at the normal time, so two peaks are inscribed. The magnitude of the first hump roughly reflects the amount of blood shunting from right to left. The height of the normal second peak tends to be low due to some dye having arrived earlier. 
Unlike left-to-right shunts, which cannot be localized by this technique, right-to-left shunts are accurately sited by serial injections. When the dye is injected in the chamber proximal to the shunt, the curves show the characteristic early appearance of dye. Beyond the shunt the dye curve is normal.

Bidirectional Shunts. Fig. 2D illustrates a bidirectional shunt, showing the combination of premature appearance of the dye due to pulmonary bypass, and delayed fall off due to pulmonary recirculation. When the right-to-left element is large, the second or normal peak is submerged, and a decision as to whether or not a right-to-left shunt is present must be made by comparing the appearance times in serial injections.

For localization, the dye curves and blood oxygen data are complementary, left-to-right shunts being localized by sampling and right-to-left shunts by the dye curves. Dye curves are more sensitive than the arterial oxygen saturation for the detection of small right-to-left shunts, and of equal sensitivity to blood oxygen determination for the detection of small left-to-right shunts.

\section{TABLE I}

The Patients Studied

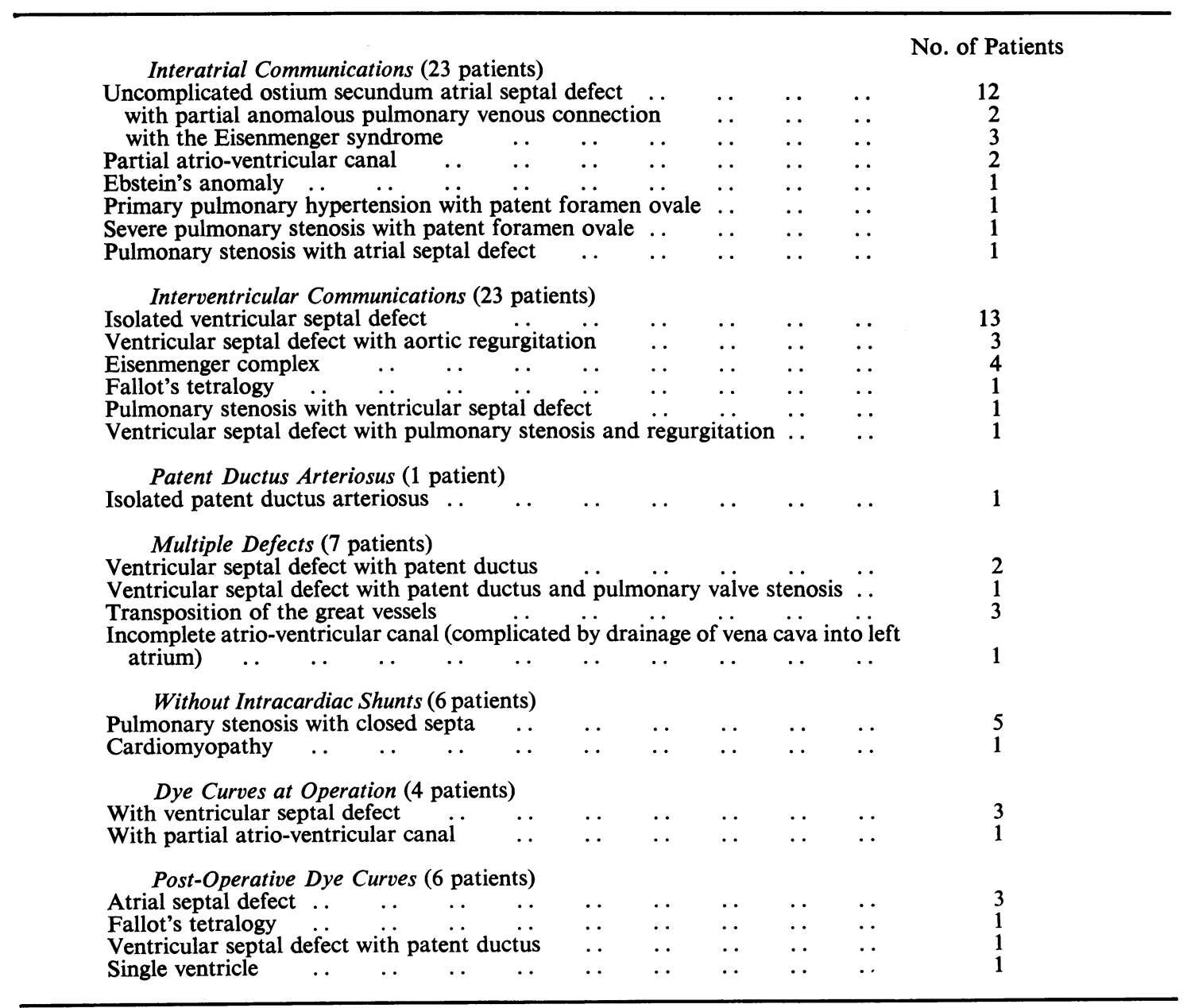




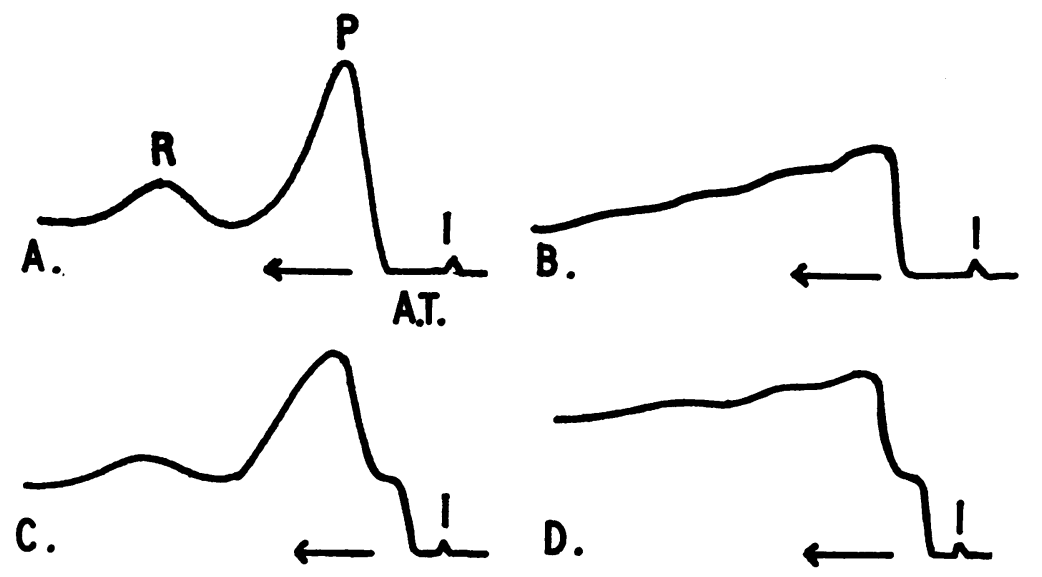

FIG. 2.-Facsimile illustrative dye curves showing (A) the normal curve, (B) left-to-right, (C) right-to-left, and (D) bidirectional shunts respectively.
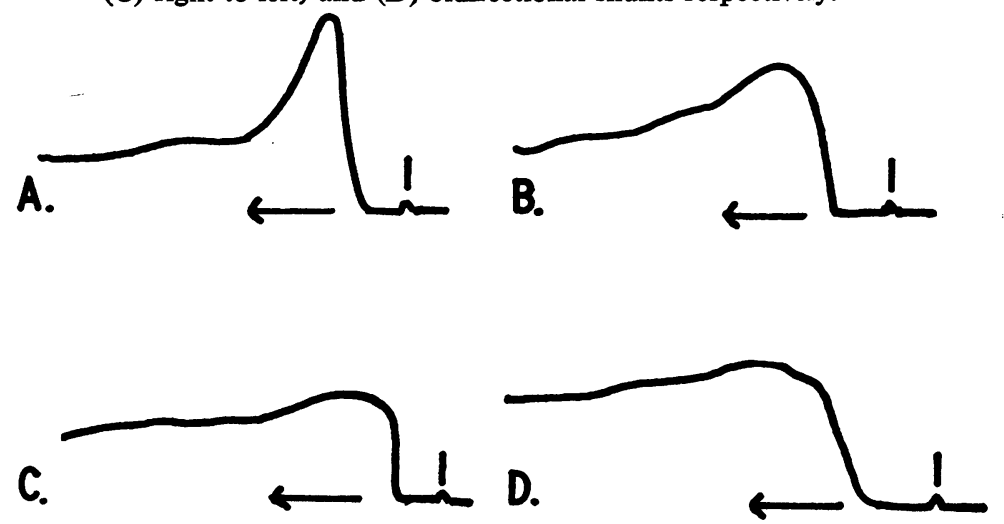

FIG. 3.-Examples of left-to-right shunts of varying size. These curves have been recorded from injections into the main pulmonary artery. Pulmonary: systemic flow ratios calculated by the Fick method were (A) $1 \cdot 7: 1$, (B) $2 \cdot 1: 1$, (C) $2 \cdot 6: 1$.
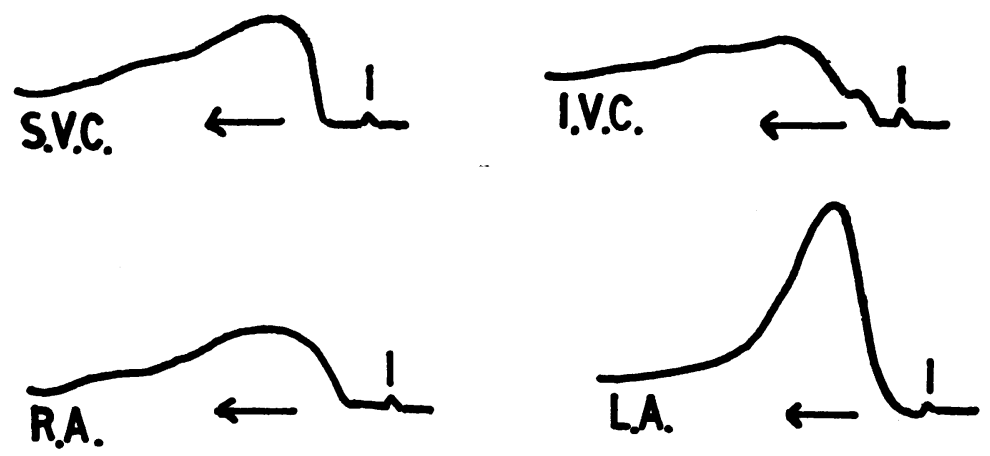

\section{ANOMALOUS PULMONARY VENOUS DRAINAGE.}

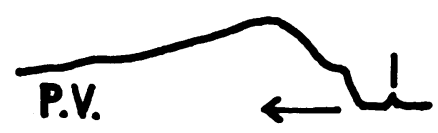

Fig. 4.-Dye curves from a patient with an atrial septal defect and an anomalous pulmonary vein.

S.V.C. \& I.V.C, superior and inferior vena cava. R.A., right atrium. L.A., left atrium. P.V., pulmonary vein. 


\section{Patients With InTER-Atrial Shunts}

Ostium Secundum Defects. Atrial septal defect presents certain important problems that are not always solved by clinical examination and right heart catheterization. These are to detect the ostium primum or atrio-ventricular canal, which must be repaired under cardio-pulmonary bypass; to distinguish the sinus venosus defect, often associated with anomalous pulmonary venous connection; and to decide whether arterial oxygen desaturation precludes operation because of pulmonary vascular disease or whether it is due to some other cause. We found dye curves to be helpful in these cases, often giving information that could not otherwise have been elicited.

Case 1, a man of 38, was thought to have a large atrial septal defect that required surgical repair. However, we were disturbed by his arterial oxygen saturation being 85 per cent despite only moderate pulmonary hypertension (pulmonary artery pressure $55 / 22 \mathrm{~mm}$. $\mathrm{Hg}$ and pulmonary vascular resistance $2 \cdot 1$ units). In view of the similarity of pulmonary and systemic arterial oxygen saturations, total anomalous pulmonary venous drainage was suspected. When there is total anomalous drainage, pulmonary venous blood reaches the left ventricle via the atrial defect so the dye appears more rapidly after injection into the right atrium than it does after injection into the pulmonary artery. This was not the case in this patient, and dye curves obtained at cardiac catheterization disposed of the possibility and explained the systemic arterial desaturation, for the curves from inferior vena cava and right atrium showed a substantial right-to-left shunt that was not seen in the curve from the superior vena cava, suggesting also that the defect was a low one. At operation a large ostium primum and ostium secundum defect, across which streaming from inferior vena cava to left atrium had occurred, was successfully closed. This patient is now well and his post-operative dye curves show no shunts.

Case 2, a woman of 54, with an atrial septal defect and kyphoscoliosis, was limited by fatigue and dyspnœea. Her arterial oxygen saturation was only 83 per cent. It was uncertain clinically whether kyphoscoliotic cor pulmonale was a factor in her disability, and would render too high the risks of operating to close the septal defect. At cardiac catheterization she was found to have a normal pulmonary vascular resistance and a pulmonary flow three times the systemic flow. Respiratory function studies disclosed mechanical ventilatory deficiency but no excessive ventilatory perfusion imbalance to account for the cyanosis. Dye curves effectively sorted out this problem. Curves from inferior vena cava and right atrium showed a right-to-left shunt. A pulmonary vein was entered and dye injection there gave rise to a curve identical with the right atrial dye curve (Fig. $4 \mathrm{~b}$ and e), suggesting the vein was draining anomalously into the right atrium. Dye injection into a normally connected pulmonary vein shows the rapid appearance time characteristic of left atrial injections. Thus dye curves in this patient indicated that the arterial desaturation was due to streaming from the inferior vana cava, suggesting a low defect, and also demonstrated anomalous pulmonary venous connection. Confirmation is lacking as this patient has not been treated surgically.

Ostium secundum defects were present in 12 patients, all of whom had clinical, radiological, and catheter evidence of a left-to-right shunt. The pulmonary/systemic blood flow ratios ranged from 1.6:1 to $4 \cdot 8: 1$ and the pulmonary vascular resistance from 0.4 to 3.0 units. Dye curves showed unequivocal evidence of left-to-right shunts in all instances. Left atrial injections were made in three patients $(1,2$, and 3$)$ and showed the early appearance time characteristic of such sites of injection (Fig. 4D).

There was a small right-to-left shunt in dye curves from the inferior vena cava and right atrium but not from the superior vena cava in three of the four patients $(1,2$, and 5) whose arterial oxygen saturation was below 94 per cent and also in two (6 and 7) whose arterial blood was 97 per cent saturated. This has been attributed to preferential streaming from inferior vena cava to left-atrium across a low defect and is unrelated to the height of the pulmonary resistance (Swan et al. 1954). A right-to-left shunt due to pulmonary hypertension shows, of course, in the dye curves from both superior and inferior cavae. The pulmonary arteriolar resistance was normal or only moderately raised in these 5 patients (range 0.5 to 3.0 units) and the three defects that have been repaired were all the ostium secundum type.

One patient (Case 3) with arterial desaturation of 87 per cent in whom dye curves showed no right-to-left shunt had extensive bronchiectasis and was rejected for surgical repair partly on this account.

Atrial Septal Defect with the Eisenmenger Syndrome. By the Eisenmenger syndrome we mean 
pulmonary hypertension of such a degree that the pulmonary resistance approximately equals the systemic resistance, this resulting in a balanced bidirectional shunt and arterial desaturation.

The diagnosis of the Eisenmenger syndrome can often be made at the bedside, but the site of the anatomical defect is often still in doubt. Right heart catheterization confirms the physiological situation but may not help to localize the shunt, for which angiocardiography or serial dye curves are required.

Case 14 was a girl, aged 6, who had had a patent ductus ligated two years previously. In the past year she had become noticeably cyanosed and was limited by fatigue and dyspnœa. Examination indicated severe pulmonary hypertension and her arterial oxygen saturation was only 72 per cent. Atrial septal defect is not often associated with a patent ductus, nor is it usually complicated by serious pulmonary hypertension at such an early age although this may have been a reaction to the combined defect. Thus it was useful to localize the site of the shunt at atrial level by means of the dye curves.

Two patients (Cases 14 and 15) with atrial septal defect and the Eisenmenger syndrome had a balanced bidirectional shunt with the calculated pulmonary resistance near or equal to the systemic resistance and accompanied by considerable arterial desaturation (less than $80 \%$ ). Caval and right atrial dye curves demonstrated the bidirectional shunt while more distal injections in the right ventricle and pulmonary artery showed only a small left-to-right shunt. The defect was thus clearly situated at atrial level (Fig. 5).

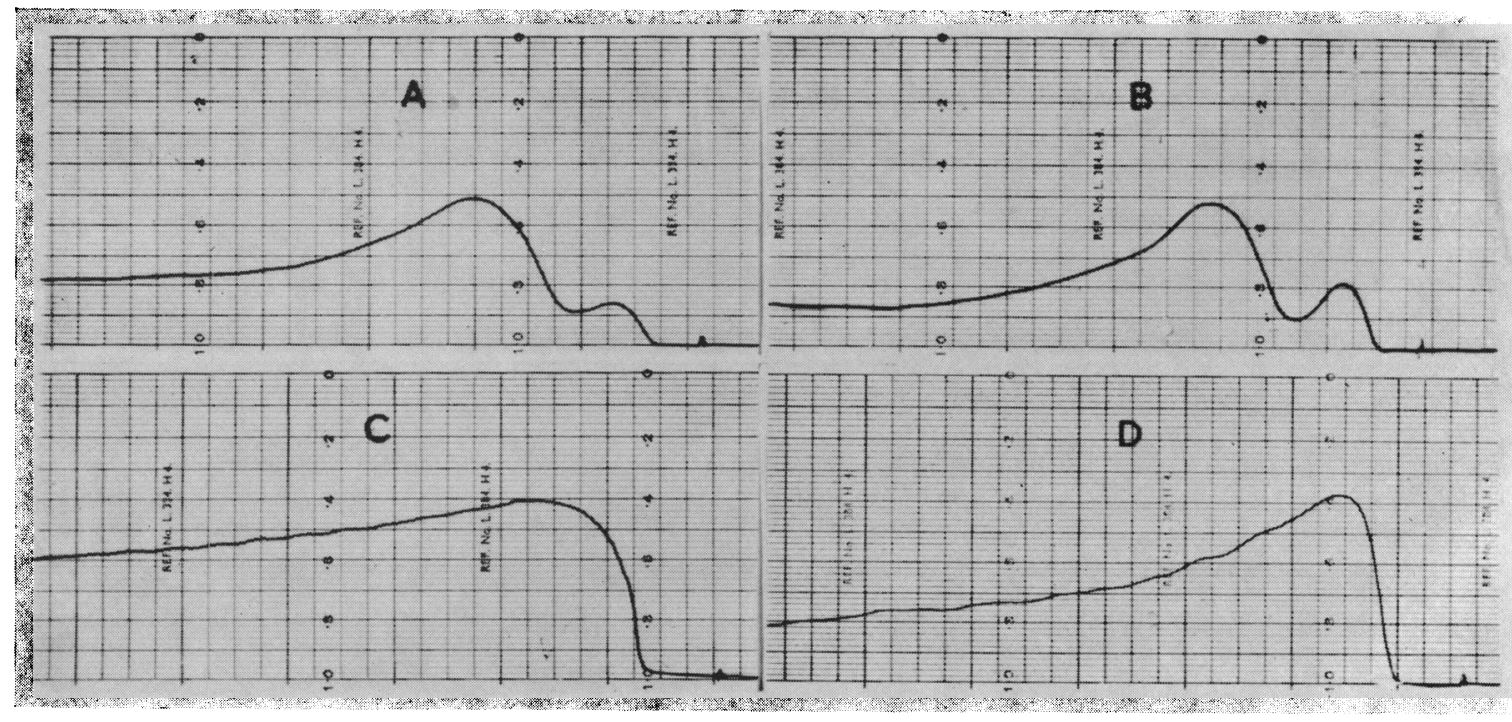

FIG. 5.-Dye curves from a patient with the Eisenmenger syndrome due to atrial septal defect, (A) superior vena caval, (B) right atrial, (C) right ventricular, and (D) pulmonary arterial injection.

Partial Atrio-Ventricular Canal (Ostium Primum Defect). There were three patients (20, 21, and 22) with partial atrio-ventricular canals, in each of whom this diagnosis was verified at operation. Dye curves showed a large left-to-right shunt in each. In one of these (Case 20) whose arterial oxygen saturation was only 75 per cent, a left superior vena cava was found at operation to enter the left atrium.

Idiopathic Pulmonary Hypertension. This is uncommon in childhood and therefore not usually suspected.

Case 18. A boy, aged 2, who had been blue from birth, was greatly disabled and deteriorating. When first seen he was deeply and symmetrically cyanosed with signs of extreme pulmonary hypertension and a very much reduced systemic flow. He was thought to have the Eisenmenger syndrome with ventricular 
septal defect and possible transposition of the great vessels. At cardiac catheterization the pulmonary arterial pressure was found to exceed the systemic pressure, and serial dye curves showed a right-to-left interatrial shunt with no opposing shunt. This was unlike the Eisenmenger syndrome in which the dye curves usually show bidirectional shunts at the appropriate level. The presence of a lone right-to-left shunt at atrial level suggested a foramen ovale rather than an atrial septal defect, and the absence of any right-to-left shunt at ventricular or pulmonary artery level excluded a ventricular defect or patent ductus. The primary lesion was, therefore, idiopathic pulmonary hypertension with secondary right-to-left shunt through a patent foramen ovale. The child died subsequently, and necropsy revealed gross right ventricular hypertrophy. The main pulmonary artery was larger than the aorta. The fossa ovalis showed valvular patency and there was no other septal defect. The ductus arteriosus was closed.

Ebstein's Anomaly. Dye curves have not proved useful in the diagnosis of Ebstein's anomaly, but injection into the superior vena cava can, by showing a bidirectional shunt, indicate that an atrial septal defect rather than a patent foramen ovale is present (Case 19).

\section{Patients With InTERVentricular Shunts}

Dye curves by our technique have little to add to the diagnosis of uncomplicated ventricular septal defect, although they are useful adjuvants when the size of the defect is under discussion with reference to its surgical closure. Shunts at ventricular level were found in 30 patients, in 16 of whom the defect was uncomplicated by other anomalies. Of these 16 patients whose pulmonary blood flows ranged from 1.4 to 2.6 times the systemic flow, 15 showed clear evidence of a left-toright shunt on the dye curves. One (Case 35) who had been diagnosed clinically as having the maladie de Roger, in whom no interventricular shunt was detectable from the blood samples at catheterization, had also normal dye curves.

Ventricular Septal Defect and Patent Ductus. The diagnosis of an associated patent ductus in cases of ventricular septal defect has found importance since the advent of intracardiac surgery, for the duct must be ligated before instituting cardiopulmonary bypass (Cleland et al., 1959). Preoperative diagnosis is difficult because the duct often seems to be functionless or carrying only a small fraction of the total shunt. Dye curves have not proved helpful here, the diagnosis being made by aortography or, latterly, by preliminary exploration at thoracotomy.

Three patients $(39,40$, and 41$)$ had both a ventricular septal defect and a patent ductus. The pulmonary blood flow in these patients ranged from 2.3 to 3.7 times the systemic flow, and the dye curves were in accord with a moderately large left-to-right shunt. In a child (Case 42) with an isolated patent ductus, whose calculated pulmonary blood flow was ten times the systemic flow, dye curves also indicated a large left-to-right shunt.

Four patients presenting as cases of Eisenmenger's syndrome were found to have interventricular shunts by means of dye curves. The dye curves in Eisenmenger's syndrome differ from the dye curves in Fallot's tetralogy in showing a bidirectional shunt. Even though the left-to-right component is small, slight skewing of the curves is usually recognizable in Eisenmenger's syndrome and this is not seen in the tetralogy, even in those acyanotic cases in whom there may be a slight left-toright shunt at rest.

Pulmonary Stenosis with Ventricular Septal Defect. The patient with severe pulmonary stenosis always poses the problem of whether an associated septal defect is present; if so, whether it is at ventricular or atrial level or not; and if at atrial level, whether there is an atrial defect or just a patent foramen ovale. Correct diagnosis has importance in order to decide whether operation should be undertaken with cardiopulmonary bypass or hypothermia.

Case 51. A girl, aged 14, had the signs of severe pulmonary stenosis, having a $4 \mathrm{~cm} . a$ wave in the neck, a long pulmonary ejection systolic murmur spilling through the aortic closure sound (A2), and a very faint, though still audible, pulmonary component of the second sound falling $0.09 \mathrm{sec}$. after A2.

The brachial artery oxygen saturation was 93 per cent and the pulmonary gradient $115 \mathrm{~mm} . \mathrm{Hg}$. There was no suggestion of a shunt from the blood oxygen data, but ear oximetry revealed that on effort there was oxygen desaturation from 94 to 83 per cent, so ventricular septal defect was suspected. Dye curves confirmed this (Fig. 6), showing a small right-to-left shunt in the curves from right atrium and right ventricle, but a 
normal dye curve from the pulmonary artery. At operation under cardiopulmonary bypass, a ventricular septal defect, $2.5 \times 2 \mathrm{~cm}$. in size, was repaired and the pulmonary infundibular stenosis resected. She is now very well.

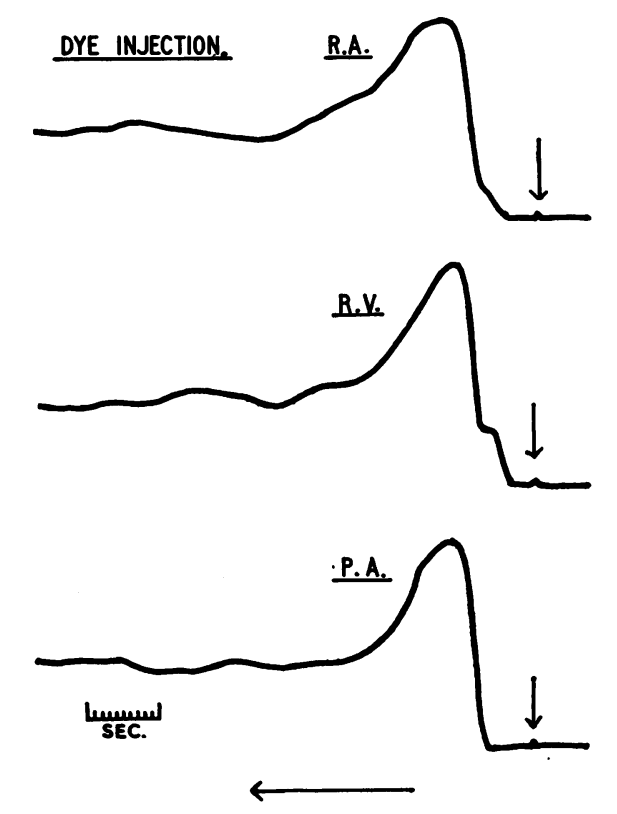

Fig. 6.-Dye curves from an acyanotic patient with severe pulmonary stenosis, showing a right-to-left shunt at ventricular level.
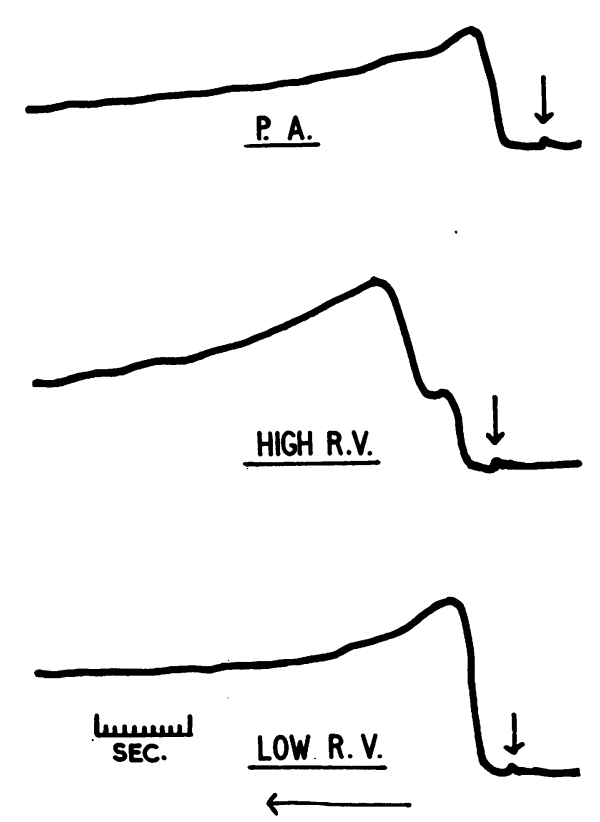

Fig. 7.-Dye curves, showing an apparent right-to-left shunt in the curve from high right ventricle, due to injection of part of the dye through a ventricular septal defect.

Transposition of the Great Vessels. Recognition of anomalous origin of the great vessels may be facilitated by dye curves, particularly in complicated situations where the exact location of the catheter tip is sometimes in doubt during catheterization. An injection of dye into the venous ventricle that appears in the rapid time characteristic of left heart injection, is diagnostic and often useful in planning the next manœuvre.

Pulmonary Stenosis. Dye curves are, of course, normal in cases of pulmonary stenosis with closed septa. The appearance time may be prolonged and the upstroke slowed by a low cardiac output in cases of extreme stenosis. In cyanosed patients, serial dye injections will localize the right-to-left shunt but cannot help to distinguish an artrial septal defect from a foramen ovale.

\section{Dye Curves Before and After Operation}

Dye curves have been used during operation to confirm successful closure of septal defects, inferior vena caval injections being made before starting the repair and again after its completion (Fig. 8). Dye curves were normal after operation in the three patients $(39,61$, and 62) in whom isolated ventricular septal defects had been repaired. The absence of a left-to-right shunt after operation was difficult to confirm in one (Case 22) after repair of a partial A-V canal: this was almost certainly due to residual regurgitation at the sutured mitral valve.

A dye injection into the S.V.C. was used as an emergency diagnostic procedure at the bedside in a patient with Fallot's tetralogy (Case 65) who rapidly went into heart failure three days after operation. It was suspected that rupture of the sutured ventricular septum had occurred and this was confirmed by dye curves which showed a large left-to-right shunt. Breakdown of the ventricular septum was confirmed at operation. 
BEFORE \& AFTER CLOSURE OF V.S.D.
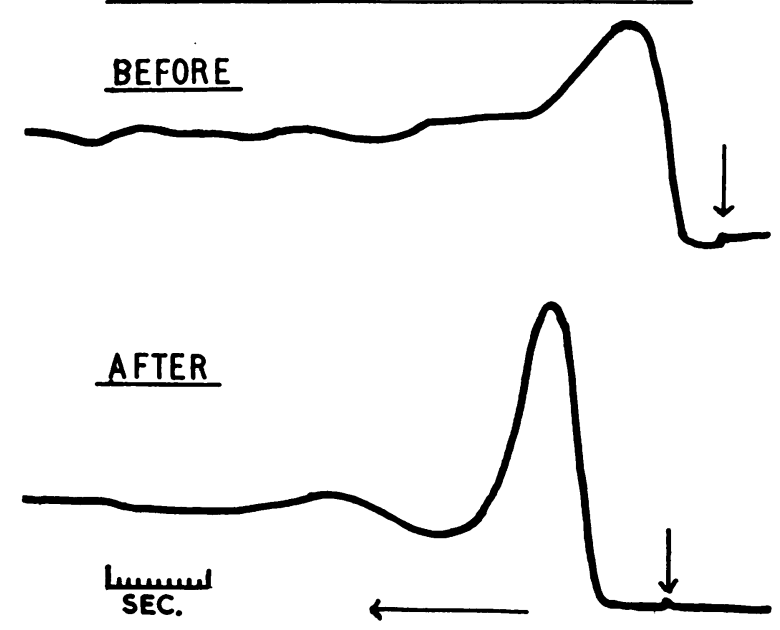

FIG. 8.-Dye curves obtained during operation and after closure of a ventricular septal defect.

Five patients $(1,41,63,64$, and 66$)$ had dye curves performed shortly after operation. Four were normal and one was abnormal. The last (Case 66) with a single ventricle had had an interventricular septal prosthesis successfully inserted. Prior to operation the physical signs were those of apparently uncomplicated ventricular septal defect but with a very large left-to-right shunt. These signs disappeared after the operation. A post-operative dye curve from the superior vena cava showed a small right-to-left but no left-to-right shunt.

\section{Difficulties ENCOUNTERED With THE TEChNIQUE}

It is necessary to know the exact location of the catheter tip and it should be the invariable rule to screen, record pressures, and take a blood sample immediately before injecting each dose of dye and to check the screening position and pressure trace again after injecting the dye. Failure to do this will inevitably lead to the faulty localization of right-to-left shunts.

Dye curves can mislead if dye is injected through defects into the left side of the heart, giving the impression of a right-to-left shunt where none in fact exists. This interpretation is avoidable if the above precautions are taken, and serial injections made.

Case 52. A man, aged 25, had a ventricular septal defect and a maldeveloped pulmonary valve with stenosis and regurgitation. Cardiac catheterization showed a right ventricular pressure of 55/12 mm., a pressure gradient of $38 \mathrm{~mm}$. across the right ventricular outflow tract, and left-to-right interventricular shunt with pulmonary blood flow five times the systemic flow. The pulmonary vascular resistance was normal. Dye injection into the right ventricular outflow tract showed, surprisingly, a right-to-left shunt also (Fig. 7). Its artefactual nature was indicated by dye curves from the right atrium, low right ventricle, and pulmonary artery which all showed only a left-to-right shunt. In fact the pressure recorded at the site of injection into the outflow tract was at systemic level, so that part of the dye had been injected into the aorta. At operation a large supra-cristal defect close under the aortic root was successfully repaired.

Toxic effects due to Coomassie blue have been negligible, consisting only in occasional vomiting usually after more than 5 or 6 dye injections. The vomitus was inky blue due to excretion of dye in the bile. Skin discoloration was absent or minimal and rapidly disappeared since there is complete clearance of dye from the body in twelve hours.

Dye was inadvertently injected into the myocardium of the left ventricle in one patient, but this is a hazard of any intracardiac injection and not peculiar to Coomassie blue dye. 
The following incident was interpreted as due to injection of dye into the myocardium, either via a Thebesian vein or due to the catheter tip becoming fixed by chordæ tendineæ.

Case 69. A child, aged 7, with an atrial septal defect that was thought to be an uncomplicated ostium secundum type needing surgical repair, was admitted for cardiac catheterization, which was performed on 10/10/58. The catheter passed early into the left atrium and an attempt was then made to enter the left ventricle. Left ventricular pressures were recorded intermittently, so it was assumed that the catheter was only just through the mitral valve. It was decided to inject dye at this site, so after withdrawing blood and then syringing through the catheter to ensure that it was free, $50 \mathrm{mg}$. of Coomassie blue was injected. Strangely, no dye curve was inscribed. Then six seconds after the injection, a small and prolonged upstroke appeared.

At this time, considerable S-T elevation appeared on the cardiogram, together with bradycardia and transient wandering atrial pacemaker. The child appeared a little distressed and complained of chest pain. After taking atrial and caval blood samples for estimation of shunt size, the procedure was terminated as rapidly as possible. Subsequently she seemed well, was afebrile, and indeed had suffered no obvious illeffect. A cardiogram (Fig. 9) later the same day did not show ST-T abnormalities and was unchanged from previous records, but one taken four days later on 14/10/58 showed deep symmetrical $T$ wave inversion in

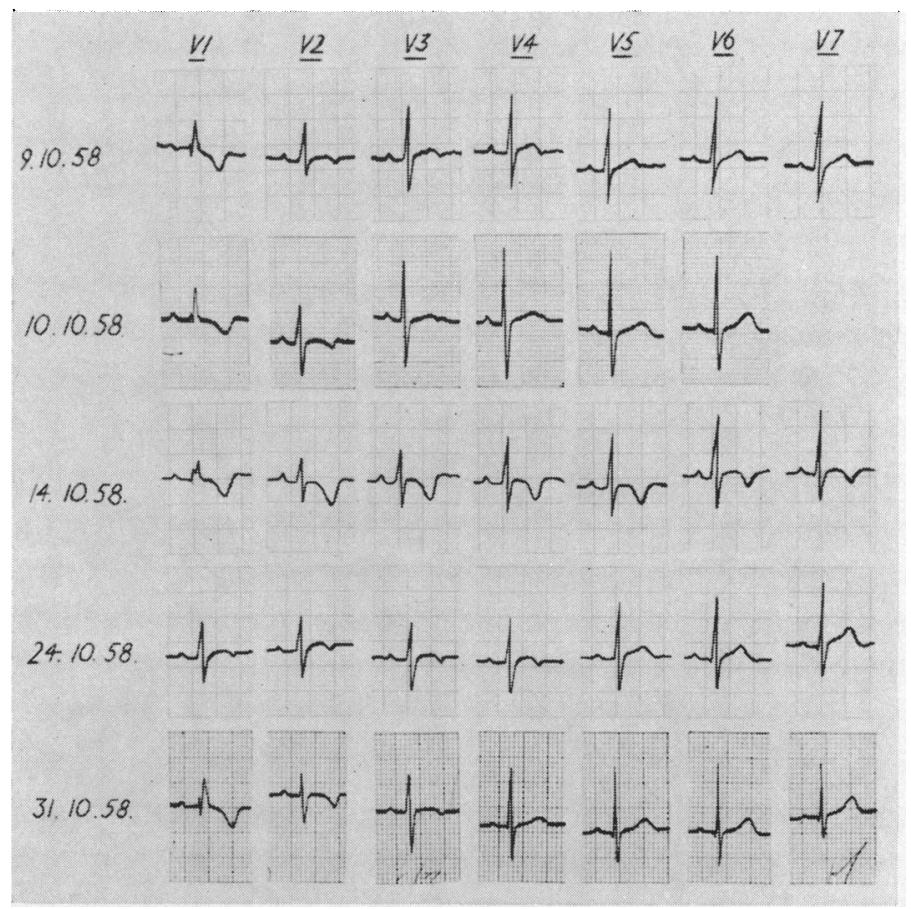

FIG. 9.-Cardiograms from a patient in whom damage to the posterior endocardial surface of the left ventricle was caused by injection of dye into the myocardium.

all the præcordial leads without abnormal Q waves. By 24/10/58, T V5-V7 were again upright but pathological upward bowing of the S-T segment in V 3 and V4 persisted. These changes were interpreted as due to damage of the posterior endocardial surface of the left ventricle. A week later, all cardiographic evidence of the episode had disappeared.

Six months later, an ostium secundum about $1.5 \mathrm{~cm}$. in diameter was closed under hypothermia. The child is now well.

\section{Discussion}

The photo-electric earpiece recorder has been used throughout to obtain the dye dilution curves. With care we have found it entirely satisfactory for the qualitative determination of the type and position of shunt. The earpiece is very sensitive to changes in position on the ear and to extraneous 
light, and it should be attached to the pinna of the ear for ten minutes before making a record. Similar results would, of course, be obtained if blood were sampled by direct arterial puncture through a photo-electric cuvette. Although the cuvette method has the advantage of greater quantitative accuracy, it has the disadvantage of the necessity of maintaining an indwelling arterial needle during the whole of the catheterization, with its attendant difficulties. It is for this reason mainly that we have explored the possibility of using the earpiece method of recording. We have had, however, little experience with the photo-electric earpiece in very young children where a cuvette might well prove better, but arterial correlation here had its own difficulties. No attempt has been made to make an accurate estimation of the size of the shunt from the curves but the cases have been divided into four main groups of shunts-none, slight, moderate, or great-by a simple inspection of the shape of the curve.

The present series of patients includes only those in whom dye was injected at the time of routine right heart catheterization, and retrograde sampling on left heart injection studies are not included. The addition of these increases the difficulty of the technique.

Coomassie blue was chosen as the dye because of its lack of toxicity even in large doses, absence of skin staining, and comparatively rapid elimination from the blood. Evans blue, on account of its undesirable staining, is probably unsuitable for multiple injections. Indigo carmine or methylene blue could both be used for this purpose. More recently the introduction of "cardio-green" has been a great advance in the technique when using a cuvette but, as it needs the infra-red cell alone for recording its passage through the ear, the use of an ear recorder tends to introduce undue pulsation.

The average dose of Coomassie blue was $0.5 \mathrm{mg}$./kilo and no trouble of any kind was experienced when giving up to ten injections at this dosage in any single patient. A slight dusky hue, sometimes apparent when multiple injections had been used, disappeared during the course of an hour or two and no evidence of skin staining was found in any patient the day after the cardiac catheterization. The technique was most reliable and failure to obtain satisfactory curves occurred only in one child due to a small ear. In some patients where there was a great variation of oxygen saturation during the respiratory cycle with consequent rhythmic variation in the base line, it was necessary to increase the dose by $1 \frac{1}{2}$ to 2 times.

Dye dilution techniques of this type are of value in the diagnosis of congenital heart disease. Used in this way, they add little to the time occupied by catheterization, and virtually nothing to the risks. Provided their limitations are recognized, they can yield much valuable information. Dye curves made with Coomassie blue dye serve as a useful screening method for left-to-right shunts, and an injection into the superior vena cava may safely be made in patients in whom cardiac catheterization is undesirable. Caval injection can also be employed to confirm obliteration of the left-to-right shunt by surgical closure of a septal defect. Intravenous injection does not always yield a reliable or accurate curve. Apart from its value as a crude quantitative screening test for left-to-right shunt, the dye curve recorded by our simple technique has its greatest value in cases with right-to-left shunts. More complicated techniques, involving left heart catheterization or simultaneous sampling from the right atrium through a cuvette, give information as to localization and magnitude of left-to-right shunts.

The simplified technique will detect a small right-to-left shunt even when the arterial oxygen saturation is normal, and will localize it with accuracy-a property of considerable value in determining the site of septal defects associated with minimal arterial desaturation. This is specially useful in cases where a left-to-right shunt at the same level is also present, for the latter is thus automatically localized. In atrial septal defect with normal pulmonary vascular resistance, a small right-to-left shunt may be the result of streaming from the inferior vena cava into the left atrium (Swan et al., 1954). By demonstrating a left-to-right shunt from the superior cava, and a bidirectional shunt from the inferior cava, the dye curve neatly reveals the cause of the arterial desaturation. 
The method is also helpful in detecting anomalous pulmonary venous drainage in atrial septal defect. The passage of the catheter into a pulmonary vein from the right atrium does not necessarily mean that the vein drains into the right atrium, for it may have been entered via the left atrium. If there is a selective right-to-left shunt from the vena cava, this will also be present when dye is injected into the pulmonary vein if the latter enters the right atrium. If there is no right-toleft shunt, then localization of the pulmonary venous drainage may depend upon the demonstration of a longer appearance time from an injection made into the pulmonary artery of the lung from which the vein drains into the right atrium (Swan and Wood, 1957). Total anomalous pulmonary venous drainage may be confirmed by the finding of a shorter appearance time when dye is injected into the right atrium than into the pulmonary artery.

The detection of anomalous origin of the great vessels may be greatly helped by dye dilution curves, for in transposition an injection into the right ventricle will have the rapid appearance time characteristic of a left ventricular injection.

In cases where arterial desaturation may be due either to pulmonary ventilation perfusion imbalance or to a right-to-left shunt, the characteristic right-to-left dye curve from right atrium, ventricle, or pulmonary artery will reveal the presence of a cardiac defect with the proviso that pulmonary arteriovenous fistulæ would be expected to cause a right-to-left shunt pattern at pulmonary artery level.

When the cardiac catheter enters the left side of the heart from the right, even more information may be obtainable. For example, in atrial septal defect when the left atrium is entered from the right, a left-to-right shunt curve will be obtained from the left atrium, but not from the left ventricle which is distal to the shunt. Thus the left-to-right shunt is localized to atrial level. When the catheter passes into the aorta from the pulmonary trunk through an aorto-pulmonary communication, injection in the ascending aorta will give a left-to-right shunt whether the fistula is a patent ductus or an aortic "window," but if the injection is made into the arch of the aorta, then a left-to-right shunt will be found if the ductus is patent, but not if there is an aortic "window," which will be proximal to the injection (Swan and Wood, 1957).

We have not had personal experience of all these conditions, but published work from the Mayo Clinic amplifies our own findings and indicates the value of the technique. We would not therefore agree with Falholt and Fabricius (1958) who considered that dye dilution curves have only a limited application in congenital heart disease. We consider, moreover, that our results show that much useful information can be gained from a technique that is simple, rapid, and virtually free from risk, and that adds considerably to the information to be obtained from catheterization of the right heart in congenital heart disease. The limitations of this simple method must be realized, however, and special care must be paid to correct recording apparatus and technique. Occasional fallacious results must be expected, especially when the catheter tip is incorrectly positioned so that dye is injected directly through a valve or septal defect into a different chamber or vessel. Careful checking of the position of the catheter is therefore essential and requires checking by fluoroscopy and manometry.

Transient endocardial damage may occur if the catheter tip is pointing into ventricular muscle, but this is a risk not peculiar to dye methods, but also applicable to the injection of any fluid rapidly into the heart. Use of Coomassie blue does not permit the blood samples to be analysed for oxygen by any photoelectric technique. In our cases the blood oxygen was obtained by the Haldane method. Alternatively dye curves may be made after the oxygen samples have been obtained.

Sets of figures with several illustrative dye curves are available on request to the authors.

Addendum. Since this paper went to press we have received reports of toxic reactions due to Coomassie blue consisting of vaso-vagal symptoms and vomiting. In most cases very large doses of over $500 \mathrm{mg}$. had been given.

\section{SUMMARY}

The application of a simple dye dilution technique to the study of congenital heart disease is described. The method employs a new dye (Coomassie blue) which is injected into the right 
side of the heart during cardiac catheterization, the dye dilution curve being recorded from an ear oximeter. The results show that this method is reliable and safe, and adds much useful information to that gained from right heart catheterization. Left-to-right shunts can be detected, and right-to-left shunts localized. Helpful data regarding anomalous origin of the great vessels and drainage of pulmonary veins have been obtained, but no attempt has been made to measure accurately the size of the shunts.

We wish to thank Miss Ena Buchanan for valuable technical help and the Department of Medical Illustration for the diagrams.

\section{REFERENCES}

Carter, S. A., Swan, H. J. C., and Wood, E. H (1959). Circulation, 19, 430.

Cleland, W. P., Beard, A. J. W., Bentall, H. H., Bishop, M. B., Braimbridge, M. W., Bromley, L. L., Goodwin, J. F., Hollman, A., Kerr, W. F., Lloyd-Jones, E. B., Melrose, D. G., and Telivuo, L. J. (1959). Brit. med. J., 2, 1369 .

Falholt, W., and Fabricius, J. (1958). Brit. Heart J., 20, 117.

Fox, I. J., Brooker, L. G. S., Hesethine, D. W., Essex, H. E., and Wood, E. H. (1957). Proc. Staff Meet. Mayo Clin., 32, 478.

Korner, P. I., and Shillingford, J. P. (1955). Clin. Sci., 14, 553.

Swan, H. J. C., Burchell, H. B., and Wood, E. H. (1954). Circulation, 10, 705.

Swan, H. J. C., and Wood, E. H. (1957). Proc. Mayo Clin., 32, 486.

- - (1957). Proc. Mayo Clin., 32, 496.

Taylor, S. H., and Shillingford, J. P. (1959). Brit. Heart J., 21, 497.

Wade, O. L., Bishop, J. M., Cumming, G., and Donald, K.'W.(1953). Brit. med. J., $2,902$.

Wood, E. H., and Garaci, J. E. (1949). J. Lab. clin. Med., 34, 387. 